\title{
NuMoon: Status of ultra high energy particle searches with LOFAR
}

\author{
S. ter Veen ${ }^{5,1 *}$, S. Buitink ${ }^{3}$, A. Corstanje ${ }^{1}$, J.E. Enriquez ${ }^{1}$, H. Falcke ${ }^{1,4,5}$, \\ J.R. Hörandel ${ }^{1,4}$, A. Nelles ${ }^{1,2}$, J.P. Rachen ${ }^{1}$, L. Rossetto ${ }^{1}$, P. Schellart ${ }^{1}$, \\ O. Scholten ${ }^{6,7}$, S. Thoudam ${ }^{1}$, T.N.G. Trinh ${ }^{6}$
}

1 Department of Astrophysics/IMAPP, Radboud University Nijmegen, P.O. Box 9010, 6500 GL Nijmegen, The Netherlands

2 Now at: Department of Physics and Astronomy, University of California Irvine, Irvine, CA 92697-4575, USA

3 Astrophysical Institute, Vrije Universiteit Brussel, Pleinlaan 2, 1050 Brussels, Belgium 4 NIKHEF, Science Park Amsterdam, 1098 XG Amsterdam, The Netherlands

5 Netherlands Institute of Radio Astronomy (ASTRON), Postbus 2, 7990 AA Dwingeloo, The Netherlands

6 KVI-CART, University Groningen, P.O. Box 72, 9700 AB Groningen, The Netherlands

7 Interuniversity Institute for High-Energy, Vrije Universiteit Brussel, Pleinlaan 2, 1050

Brussels, Belgium

E-mail: veeneastron.nl

\begin{abstract}
The lunar Askaryan technique is one of the few ways to obtain a large enough collecting area to detect ultra high energy cosmic rays and neutrinos at the highest end of the known spectrum, above $10^{21} \mathrm{eV}$. The flux of these particles is unknown, but if they are found they either point back to the best cosmic accelerators or they can be the products of the decay of exotic particles such as cosmic strings. Using the lunar Askaryan technique means searching for radio signals created by a particle shower that is induced when an ultra high energy particle collides with the Moon. The large collecting area that the Moon offers can especially be used at frequencies between 100-200 $\mathrm{MHz}$, where the radiation is spread out over a wider angle and thus more of the lunar surface can be used for a possible detection. The NuMoon project therefore observes the Moon at these frequencies to search for nanosecond pulses. A first project with the Westerbork Synthesis Radio Telescope has placed the most stringent upper limits on the flux of ultra high energy cosmic rays and neutrinos. The next step is to observe with LOFAR, currently one of the most sensitive low frequency telescopes. In this contribution I will present the status and plans of the project.
\end{abstract}

The 34th International Cosmic Ray Conference,

30 July- 6 August, 2015

The Hague, The Netherlands

\footnotetext{
* Speaker.
} 


\section{Introduction}

Two of the main open questions in astroparticle physics are: to what energy can particles be accelerated and what is the origin of the most energetic particles? To answer these questions it is required to measure the spectrum to higher and higher energies. An alternative source of particles with the highest energies would be the decay from heavy exotic particles, such as predicted by extensions to the standard model. The highest energy cosmic rays detected so far have energies of more than $10^{20} \mathrm{eV}$ [1], but it is not clear if this is at the fundamental acceleration limit, or because of the GZK effect [2,3]. In the latter case it is possible to search for ultra high energy neutrinos instead, that would have been formed in the GZK interactions. Unfortunately, both of these particles are very rare at less than 1 per $\mathrm{km}^{2}$ per century. And therefore detection requires monitoring a large quantity of air [4], ice [5, 6] or the Moon to detect such particles.

\section{The lunar Askaryan technique}

When a high energy particle interacts with the Moon, it creates a cascade of particles. The creation and annihilation of particles in this shower creates a coherent radio signal at frequencies of more than $3 \mathrm{GHz}$ down to below $100 \mathrm{MHz}$ that can be observed with a radio telescope. This was first suggested by [7]. The source of the radiation is also known as the Askaryan effect [8, 9], and therefore this method is known as the lunar Askaryan technique. At gigahertz frequencies the radiation is beamed, giving geometrical constraints on particle direction with respect to the Moon surface. At $200 \mathrm{MHz}$ frequency, the radiation is spread out over a larger angle, increasing the effective aperture for particle detection [10], at the cost of a lower signal. To have the highest aperture, the NuMoon project observes at frequencies between 100 and $200 \mathrm{MHz}$. A first project with the Westerbork Synthesis Radio Telescope has produced the most stringent upper limits for neutrinos above $4 \times 10^{22} \mathrm{eV}$ [11] and cosmic rays above $10^{22} \mathrm{eV}$ [12]. The next step is to observe with the Low Frequency Array (LOFAR [13]), a new telescope that has been built in the Netherlands over the last years and is one of the largest low frequency telescopes currently available.

\section{LOFAR implementation of the lunar Askaryan technique}

The lunar Askaryan method requires a sensitive radio telescope to be pointed at the Moon and be able to detect signals of only a few tens of nanoseconds, while rejecting any false positives. This is a demanding operation for a low frequency radio telescope, but for LOFAR it builds upon different aspects of the system that are used for other types of observations. This section describes the requirements of the method as applied to the LOFAR radio telescope [13] with examples from different sciences cases.

To detect a weak signal, the antennas need to be added coherently in the direction of the Moon. This process is called beam forming and is used for high time resolution astronomy such as the study of pulsars and solar emission. Beam forming with LOFAR is a two stage process. First the 48 antennas within a station are added together to form a sub-array pointing, separately for each polarization. To do this a poly phase filter and Fourier transform are applied to the data to create 

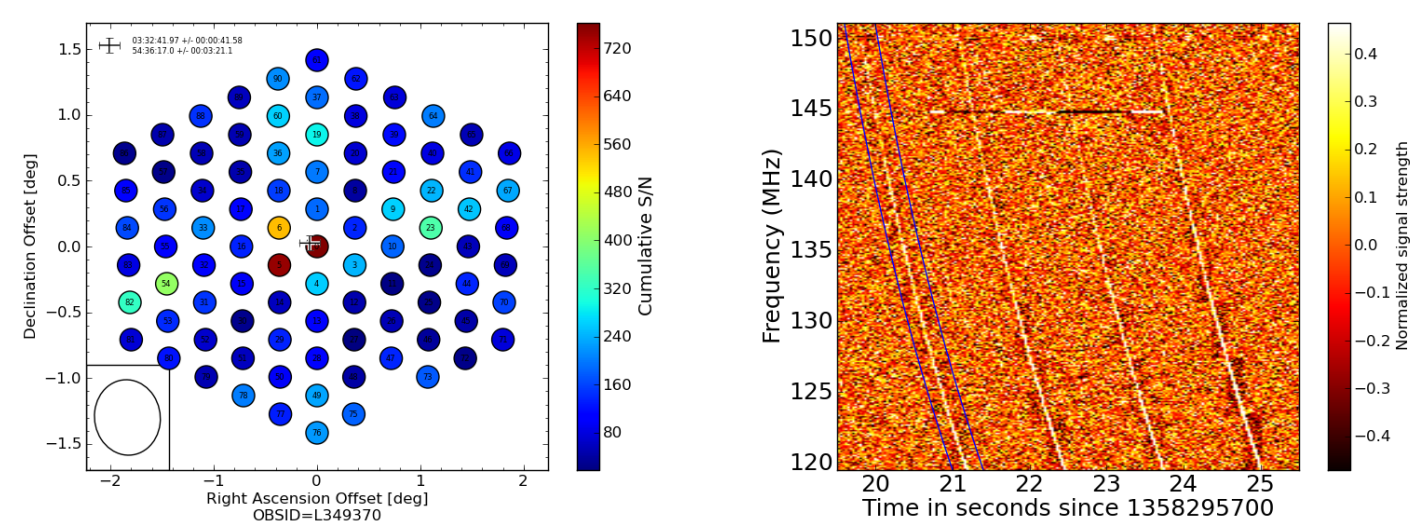

Figure 1: Left: Example of a multi-beam observation: Pulsar B0329+54, showing signal to noise ratio in all beams. The highest signal is found at the centre. Right: Dispersed pulses from pulsar B0834+26.

512 frequency channels or subbands. The geometrical delay and any calibration values are added as a complex weight to each subband of each antenna. These are then summed together to form the beam. A maximum of 488 subbands can be selected ${ }^{1}$ to do the beam forming in a certain direction. In this case 488 consecutive subbands are beam formed towards the Moon in the range 110-190 MHz. The beam is wide enough to cover the complete Moon. The second step is to combine the different stations. For this only the 24 core stations are selected. There are three reasons for this. One is that they are connected to a single clock. Therefore coherent addition is easier, because there are no clock drift. The second reason is that the beam size depends on the distance between the stations. The further they are apart, the smaller the beam size. To cover a significant fraction of the lunar surface too many beams are required if the stations are too far apart. The third reason is that the core stations see a similar part of the sky, which makes ionospheric corrections relatively easy.

The beam size of combining the core stations is smaller than the Moon, at around 4 arc minutes. Therefore 50 beams are used to cover the Moon surface. In pulsar observations, such a setup is already used successfully to determine the position of newly discovered sources [14]. An example for the long known pulsar B0329+54 is shown in figure 1, this time with 6 of the 24 core stations, and therefore a larger beam size. With NuMoon we can do the same, and also use an anticoincidence trigger to detect instrumental problems or (terrestrial) interference, that would appear in multiple beams. For example, a signal because of a malfunction in one of the station beams may appear in all tied array beams. For a real signal we have to take into account the relative signal in other beams for signals that come from the Moon. The pulsar in figure 1 is for example detectable in all beams, but the signal to noise ratio is different. An anti-coincidence signal was also previously applied successfully at the WSRT $[15,11]$ and Parkes [16] telescopes.

To observe and find pulsars a correction is required for the dispersion in arrival time for the different frequencies, because of the different refraction index for different frequencies because of free electrons in the interstellar medium, as shown in Figure 1. A similar effect appears for the ionosphere. The duration of this effect is of course much smaller, but it is still significant for the

\footnotetext{
${ }^{1}$ when using 8-bits per sample
} 


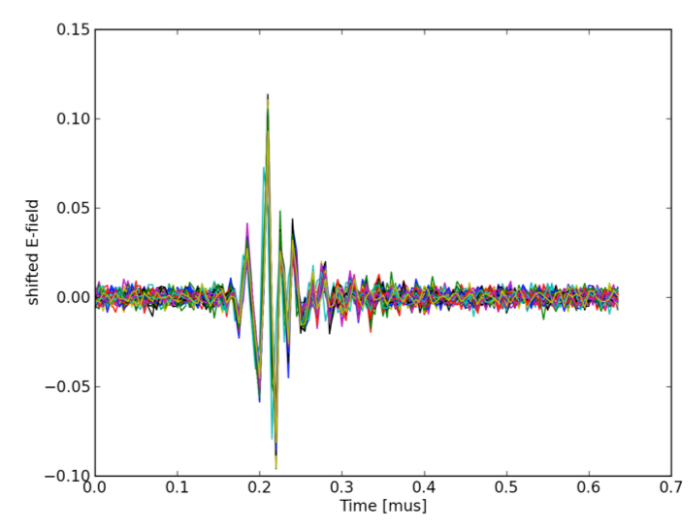

Figure 2: Raw data of the antennas of one polarisation of one station showing the radio signal of an extensive air shower from a cosmic ray. The data has been aligned in time for the arrival time of the pulses.

duration of the pulses. An accurate correction is required. Therefore we try to predict or measure the current Total Electron Content (TEC) value, and also dedisperse at a few surrounding values. The inner stations are usually under the same ionospheric bubble, such that we do not have to apply different values for the different stations.

The signal from a cosmic particle is of the order of $10 \mathrm{~ns}$. The normal time resolution after beam forming is at least 5 microseconds. Therefore we need to go back to the full time resolution of $5 \mathrm{~ns}$. This was lost by the poly phase filter applied in the station beam forming. A poly phase filter inversion needs to be applied to data to restore this time resolution. The effect of this processing step is detailed in [17]. But since that publication, more subbands have been made available in the LOFAR system, such that all RFI-free subbands can be selected in this process.

There is another project that deals with such short signals with LOFAR. This is the detection of very high energy cosmic rays with energies of $10^{16.5} \mathrm{eV}-10^{17.5} \mathrm{eV}$ and higher [18]. These create extensive air showers in the Earths atmosphere. In this case the trigger comes from a particle detector array (LORA[19]). The radio signal comes from the charged particles in this air shower, and its interaction with the earth's magnetic field. The signal is read out from buffers for each individual dipole. An example of such a signal is shown in figure 3. This project is collecting data and can detect the composition of the primary particle [20]. The first results have been published in several journals $[21,22,23,24]$ and are also presented at this conference.

Each of the 50 beams will be searched for short signals. For this the power is added for $\mathrm{N}$ consecutive samples using a sliding window. This was investigated in [17], but needs to be revised now that more subbands are available, and depending on how well the TEC value can be determined. If a signal is found, a read out is requested for the same buffers used for the extensive air shower experiment. Data is requested not only from the core stations used for triggering, but also from the remote and international stations that are further away and are more sensitive. This data can be used to verify the signal, using the higher signal to noise ratio and a precise localisation to show that it is not local and not instrumental but actually originates from the Moon. It is then also used to reconstruct where on the Moon is was emitted, such that the direction of the signal can be linked to a position on the sky and thus to the source of ultra high energy particles. 

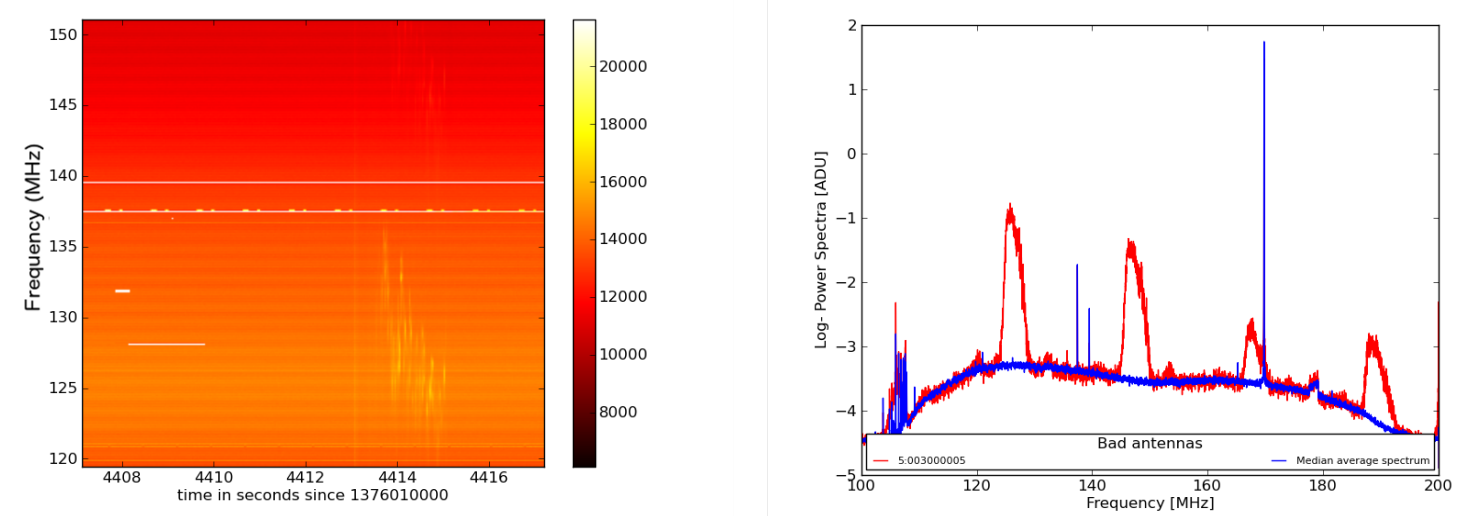

Figure 3: Left: The dynamic spectrum showing a peculiar event detected with the FRATS real-time trigger. Right: The spectrum of one misbehaving antenna [red] compared to the median spectra of all antennas in the LOFAR station [blue]. This antenna caused the signal on the left.

This is not the only project that searches for a signal in real time on LOFAR beam formed data and then reads out the buffers to analyse the signal. The FRATS (Fast Radio Transient Search $[25,26])$ project uses millisecond time-resolution data to search for dispersed pulses. These can come from pulsars, but more interestingly may be Fast Radio Bursts (FRBs [27]), highly dispersed pulses that are likely originating from outside our galaxy. Apart from detecting several known pulsars, the FRATS project has also identified a broken antenna and a solar burst through the buffered data [26]. This shows the use of the buffered data for identification, and that such a realtime trigger project is feasible.

\subsection{Status of the project}

As described above, many of the requirements for the NuMoon project are ready. However, there are still some essential parts missing, such as the online implementation of the inverse poly phase filter and the trigger itself. The next step is to test and try these steps off-line. The upcoming off-line cluster for LOFAR with an increased bandwidth will be very useful for this, by being able to record close to 50 beams to disk, instead of 7 currently. The sensitivity for a total of one week observing time, according to [17] are presented in figure 4.

\section{Conclusion}

The lunar Askaryan technique is one of the few ways to obtain enough collecting area to detect ultra high energy cosmic rays and neutrinos. It does this by observing the Moon with a radio telescope. At low frequencies $(150 \mathrm{MHz})$ the effective area is even larger, because the radiation spreads out. A project with the WSRT resulted in the most stringent limits at energies above $10^{22}$ - $10^{23} \mathrm{eV}$. The more powerful LOFAR, will be able to lower the energy threshold. To do this it requires multiple, very high time resolution beams to be pointed at the Moon. On each beam a trigger runs to detect pulses of only a few 10s of nanoseconds. A coincidence trigger can be used to distinguish between signals and interference. To verify / falsify triggers, the data from 

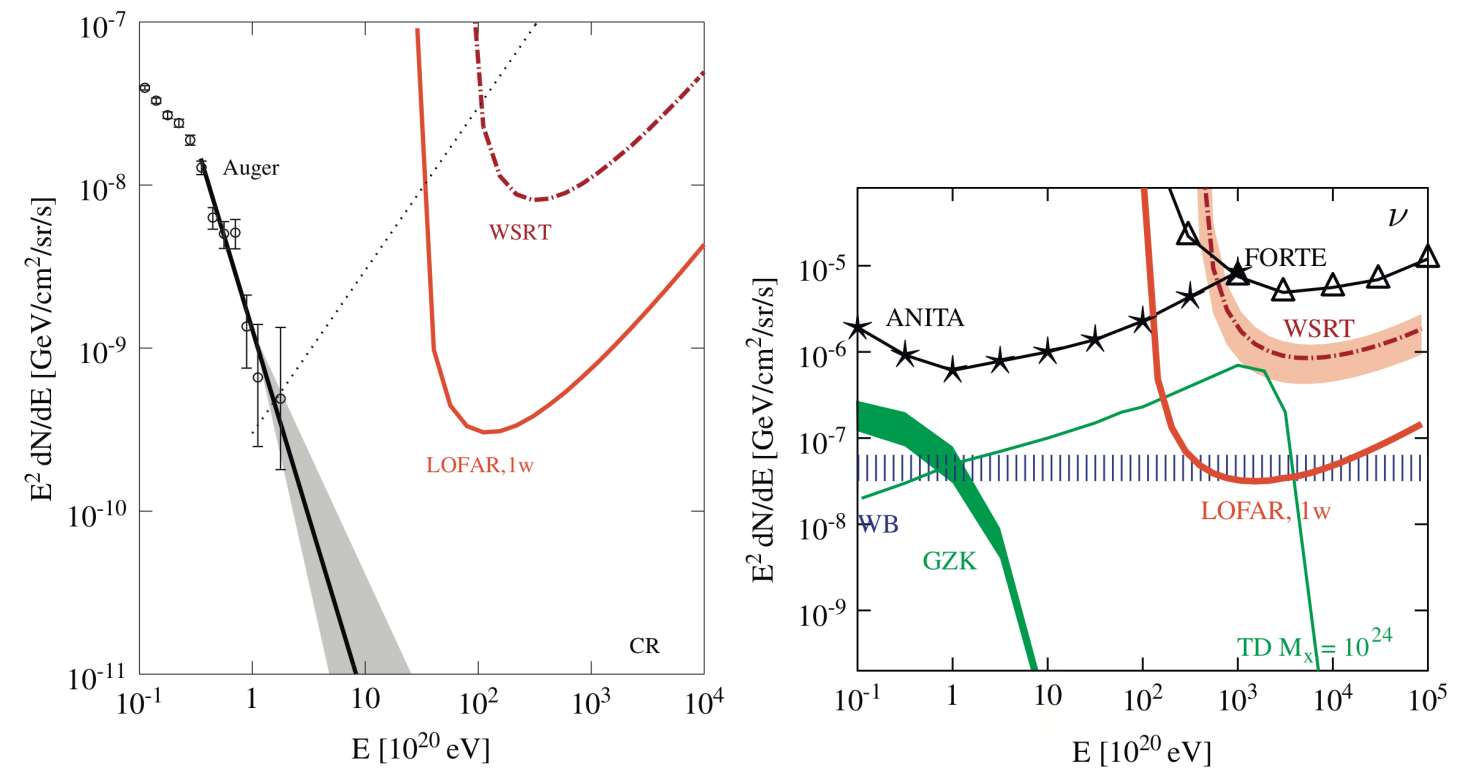

Figure 4: Sensitivity in a one week LOFAR observation is compared with data from other experiments (WSRT, Auger, ANITA, FORTE), neutrinos expected from the GZK cut-off, the Waxmann-Bahcall limit (WB) and a top-down model (TD). (left) cosmic rays; (right) neutrinos.

the individual antennas can be used to reconstruct the signal off-line. The project has not been able to take data yet, because essential parts are missing in the telescope software, such as the inversion to time domain. However, many parts of the system have been demonstrated by other scientific applications, such as multi-beaming to find the position of pulsars, high time resolution data processing to detect cosmic rays air showers, and on-line detection and off-line verification to search for dispersed millisecond signals from pulsars and fast radio bursts.

\section{Acknowledgement}

The LOFAR cosmic ray key science project acknowledges funding from an Advanced Grant of the European Research Council (FP/2007-2013) / ERC Grant Agreement n. 227610. The project has also received funding from the European Research Council (ERC) under the European Union's Horizon 2020 research and innovation programme (grant agreement No 640130). We furthermore acknowledge financial support from FOM, (FOM-project 12PR304) and NWO (VENI grant 639041-130). AN is supported by the DFG (research fellowship NE 2031/1-1).

LOFAR, the Low Frequency Array designed and constructed by ASTRON, has facilities in several countries, that are owned by various parties (each with their own funding sources), and that are collectively operated by the International LOFAR Telescope foundation under a joint scientific policy.

\section{References}

[1] A. Aab and the Pierre Auger Collaboration, Searches for anisotropies in the arrival directions of the highest energy cosmic rays detected by the pierre auger observatory, The Astrophysical Journal $\mathbf{8 0 4}$ 
(2015), no. 115 .

[2] K. Greisen, End to the Cosmic-Ray Spectrum?, Physical Review Letters 16 (Apr., 1966) 748-750.

[3] G. T. Zatsepin and V. A. Kuz'min, Upper Limit of the Spectrum of Cosmic Rays, Soviet Journal of Experimental and Theoretical Physics Letters 4 (Aug., 1966) 78.

[4] A. Haungs and JEM-EUSO Collaboration, Physics Goals and Status of JEM-EUSO and its Test Experiments, ArXiv e-prints (Apr., 2015) [arXiv: 1504.0259$].$

[5] P. W. Gorham, P. Allison, B. M. Baughman, J. J. Beatty, K. Belov, D. Z. Besson, S. Bevan, W. R. Binns, C. Chen, P. Chen, J. M. Clem, A. Connolly, M. Detrixhe, D. De Marco, P. F. Dowkontt, M. DuVernois, E. W. Grashorn, B. Hill, S. Hoover, M. Huang, M. H. Israel, A. Javaid, K. M. Liewer, S. Matsuno, B. C. Mercurio, C. Miki, M. Mottram, J. Nam, R. J. Nichol, K. Palladino, A. Romero-Wolf, L. Ruckman, D. Saltzberg, D. Seckel, G. S. Varner, A. G. Vieregg, and Y. Wang, Observational constraints on the ultrahigh energy cosmic neutrino flux from the second flight of the anita experiment, Phys. Rev. D 82 (Jul, 2010) 022004.

[6] P. W. Gorham, P. Allison, B. M. Baughman, J. J. Beatty, K. Belov, D. Z. Besson, S. Bevan, W. R. Binns, C. Chen, P. Chen, J. M. Clem, A. Connolly, M. Detrixhe, D. De Marco, P. F. Dowkontt, M. DuVernois, E. W. Grashorn, B. Hill, S. Hoover, M. Huang, M. H. Israel, A. Javaid, K. M. Liewer, S. Matsuno, B. C. Mercurio, C. Miki, M. Mottram, J. Nam, R. J. Nichol, K. Palladino, A. Romero-Wolf, L. Ruckman, D. Saltzberg, D. Seckel, R. Y. Shang, G. S. Varner, A. G. Vieregg, and Y. Wang, Erratum: Observational constraints on the ultrahigh energy cosmic neutrino flux from the second flight of the anita experiment [phys. rev. d 82022004 (2010)], Phys. Rev. D 85 (Feb, 2012) 049901.

[7] R. D. Dagkesamanskij and I. M. Zheleznykh, Radio-astronomy method for detecting neutrinos and other elementary particles of superhigh energy., Soviet Journal of Experimental and Theoretical Physics Letters 50 (Sept., 1989) 259-261.

[8] G. A. Askar'yan, Coherent Radio Emission from Cosmic Showers in Air and in Dense Media, Soviet Journal of Experimental and Theoretical Physics 21 (Sept., 1965) 658.

[9] G. A. Askaryan, Excess Negative Charge of the Electron-Photon Shower and Coherent Radiation Originating from It. Radio Recording of Showers under the Ground and on the Moon, Journal of the Physical Society of Japan Supplement 17 (1962) C257.

[10] O. Scholten, J. Bacelar, R. Braun, A. G. de Bruyn, H. Falcke, B. Stappers, and R. G. Strom, Optimal radio window for the detection of Ultra-High Energy cosmic rays and neutrinos off the moon, Astroparticle Physics 26 (Oct., 2006) 219-229, [astro-ph/ 0609179 ].

[11] S. Buitink, O. Scholten, J. Bacelar, R. Braun, A. G. de Bruyn, H. Falcke, K. Singh, B. Stappers, R. G. Strom, and R. A. Yahyaoui, Constraints on the flux of ultra-high energy neutrinos from Westerbork Synthesis Radio Telescope observations, Astronomy \& Astrophysics 521 (Oct., 2010) A47, [arXiv:1004.0274].

[12] S. Ter Veen, S. Buitink, H. Falcke, C. W. James, M. Mevius, O. Scholten, K. Singh, B. Stappers, and K. D. de Vries, Limit on the ultrahigh-energy cosmic-ray flux with the Westerbork synthesis radio telescope, Physical Review D 82 (Nov., 2010) 103014, [arXiv:1010.6061].

[13] M. P. van Haarlem, M. W. Wise, et al., LOFAR: The LOw-Frequency ARray, Astronomy \& Astrophysics 556 (Aug., 2013) A2, [arXiv:1305.3550].

[14] T. Coenen et al., The LOFAR pilot surveys for pulsars and fast radio transients, Astronomy \& Astrophysics 570 (Oct., 2014) A60, [arXiv: 1408.0411 ]. 
[15] O. Scholten, S. Buitink, J. Bacelar, R. Braun, A. G. de Bruyn, H. Falcke, K. Singh, B. Stappers, R. G. Strom, and R. Al Yahyaoui, Improved Flux Limits for Neutrinos with Energies above $10^{22} \mathrm{eV} \mathrm{from}$ Observations with the Westerbork Synthesis Radio Telescope, Physical Review Letters 103 (Nov., 2009) 191301, [arXiv:0910.4745].

[16] J. D. Bray, R. D. Ekers, P. Roberts, J. E. Reynolds, C. W. James, C. J. Phillips, R. A. McFadden, R. J. Protheroe, M. Aartsen, and J. Alvarez-Muñiz, Status and strategies of current LUNASKA lunar Cherenkov observations with the Parkes radio telescope, Nuclear Instruments and Methods in Physics Research A 662 (Jan., 2012) 95, [arXiv: 1010.1997$].$

[17] K. Singh, M. Mevius, O. Scholten, et al., Optimized trigger for ultra-high-energy cosmic-ray and neutrino observations with the low frequency radio array, Nuclear Instruments and Methods in Physics Research A 664 (Feb., 2012) 171-185, [arXiv:1108.5745].

[18] P. Schellart, A. Nelles, et al., Detecting cosmic rays with the LOFAR radio telescope, Astronomy \& Astrophysics 560 (Dec., 2013) A98, [arXiv:1311.1399].

[19] S. Thoudam et al., Lora: A scintillator array for \{LOFAR\} to measure extensive air showers, Nuclear Instruments and Methods in Physics Research Section A: Accelerators, Spectrometers, Detectors and Associated Equipment 767 (2014), no. 0339 - 346.

[20] S. Buitink, A. Corstanje, J. E. Enriquez, H. Falcke, J. R. Hörandel, T. Huege, A. Nelles, J. P. Rachen, P. Schellart, O. Scholten, S. ter Veen, S. Thoudam, and T. N. G. Trinh, Method for high precision reconstruction of air shower $X_{\max }$ using two-dimensional radio intensity profiles, Physical Review $D$ 90 (Oct., 2014) 082003, [arXiv: 1408 . 7001 ].

[21] P. Schellart et al., Polarized radio emission from extensive air showers measured with LOFAR, JCAP 10 (Oct., 2014) 14, [arXiv:1406.1355].

[22] A. Nelles, P. Schellart, and the LOFAR collaboration, Measuring a Cherenkov ring in the radio emission from air showers at 110-190 MHz with LOFAR, Astroparticle Physics 65 (2015) 11-21, [arXiv:1411.6865].

[23] A. Corstanje, P. Schellart, et al., The shape of the radio wavefront of extensive air showers as measured with LOFAR, Astroparticle Physics 61 (Feb., 2015) 22-31, [arXiv: 1404 . 3907 ].

[24] A. Nelles, S. Buitink, A. Corstanje, J. E. Enriquez, H. Falcke, J. R. Hörandel, J. P. Rachen, L. Rossetto, P. Schellart, O. Scholten, S. ter Veen, S. Thoudam, and T. N. G. Trinh, The radio emission pattern of air showers as measured with LOFAR - a tool for the reconstruction of the energy and the shower maximum, JCAP 5 (May, 2015) 18, [arXiv:1411.7868].

[25] S. ter Veen, P. Schellart, H. Falcke, and Falcke, FRATs: Searching for Fast Radio Transients in Real Time with LOFAR, in IAU Symposium (E. Griffin, R. Hanisch, and R. Seaman, eds.), vol. 285 of IAU Symposium, pp. 411-413, Apr., 2012.

[26] S. ter Veen, Searching for Fast Radio Transients with LOFAR. PhD thesis, Radboud Universiteit Nijmegen, Submitted 2015.

[27] D. Thornton, B. Stappers, M. Bailes, B. Barsdell, S. Bates, N. D. R. Bhat, M. Burgay, S. Burke-Spolaor, D. J. Champion, P. Coster, N. D’ Amico, A. Jameson, S. Johnston, M. Keith, M. Kramer, L. Levin, S. Milia, C. Ng, A. Possenti, and W. van Straten, A Population of Fast Radio Bursts at Cosmological Distances, Science 341 (July, 2013) 53-56, [arXiv: 1307.1628 ]. 